\title{
Joint density of electronic states for one isolated single-wall carbon nanotube studied by resonant Raman scattering
}

\section{Citation}

Jorio, A., A. G. Souza Filho, G. Dresselhaus, M. S. Dresselhaus, R. Saito, J. H. Hafner, C. M. Lieber, F. M. Matinaga, M. S. S. Dantas, and M. A. Pimenta. 2001. "Joint Density of Electronic States for One Isolated Single-Wall Carbon Nanotube Studied by Resonant Raman Scattering." Physical Review B 63 (24). https://doi.org/10.1103/physrevb.63.245416.

\section{Permanent link}

http://nrs.harvard.edu/urn-3:HUL.InstRepos:41417229

\section{Terms of Use}

This article was downloaded from Harvard University's DASH repository, and is made available under the terms and conditions applicable to Other Posted Material, as set forth at http:// nrs.harvard.edu/urn-3:HUL.InstRepos:dash.current.terms-of-use\#LAA

\section{Share Your Story}

The Harvard community has made this article openly available.

Please share how this access benefits you. Submit a story.

\section{Accessibility}




\title{
Joint density of electronic states for one isolated single-wall carbon nanotube studied by resonant Raman scattering
}

\author{
A. Jorio, ${ }^{1}$ A. G. Souza Filho, ${ }^{1,4}$ G. Dresselhaus, ${ }^{3}$ M. S. Dresselhaus, ${ }^{1,2}$ R. Saito, ${ }^{5}$ J. H. Hafner, ${ }^{6}$ C. M. Lieber, ${ }^{6}$ \\ F. M. Matinaga, ${ }^{7}$ M. S. S. Dantas, ${ }^{7}$ and M. A. Pimenta ${ }^{7}$ \\ ${ }^{1}$ Department of Physics, Massachusetts Institute of Technology, Cambridge, Massachusetts 02139-4307 \\ ${ }^{2}$ Department of Electrical Engineering and Computer Science, Massachusetts Institute of Technology, \\ Cambridge, Massachusetts 02139-4307 \\ ${ }^{3}$ Francis Bitter Magnet Laboratory, Massachusetts Institute of Technology, Cambridge, Massachusetts 02139-4307 \\ ${ }^{4}$ Departamento de Física, Universidade Federal do Ceará, Fortaleza - CE, 60455-760 Brazil \\ ${ }^{5}$ Department of Electronic-Engineering, University of Electro-Communications, Tokyo, 182-8585 Japan \\ ${ }^{6}$ Department of Chemistry, Harvard University, Cambridge, Massachusetts 02138 \\ ${ }^{7}$ Departamento de Física, Universidade Federal de Minas Gerais, Belo Horizonte - MG, 30123-970 Brazil
}

(Received 21 December 2000; published 6 June 2001)

\begin{abstract}
Resonant Raman scattering (RRS) measurements made with a tunable laser provide a highly reliable technique to study the shape of the joint density of electronic states (JDOS) of isolated single-wall carbon nanotubes (SWNTs). RRS can be used to determine the energy value for the one-dimensional (1D) van Hove singularities of a SWNT with a precision better than $5 \mathrm{meV}$, thereby providing important information that could be used for subsequent measurements on this same SWNT. With RRS, the measured width of the JDOS is on the order of $\sim 0.1-1.0 \mathrm{meV}$, further demonstrating that SWNTs really provide a remarkably good model for $1 \mathrm{D}$ mesoscopic systems.
\end{abstract}

DOI: 10.1103/PhysRevB.63.245416

PACS number(s): 78.30.Na, 78.66.Tr

One dimensional (1D) systems are predicted to exhibit very interesting physical properties arising from the quantum confinement of electrons in the 1D lattice. Due to this quantum confinement, the density of electronic states (DOS) in 1D systems exhibits van Hove singularities, where, in the case of a perfect infinite 1D lattice, the DOS goes to infinity for well-defined energy values. The confinement of a large number of electronic states into a single energy value results in striking physical properties, such as extremely high optical absorption and emission, enhanced thermoelectric power, quantized electronic conductivity, etc. Applications based on these properties could lead to important new technological devices.

Since their first observation in 1993, ${ }^{1,2}$ single-wall carbon nanotubes (SWNTs) have attracted much attention in the physics community, because of the remarkable electronic and mechanical properties theoretically predicted for these 1D mesoscopic systems. ${ }^{3,4}$ Although experiments, such as resonant Raman scattering (RRS) ${ }^{5-7}$ and optical absorption, ${ }^{8}$ have shown the presence of quantum confinement effects in the DOS of SWNTs, it is very difficult to properly characterize the DOS experimentally, because of the difficulty of making detailed measurements on a single molecule $\sim 1 \mathrm{~nm}$ in diameter. Most of the experimental studies have been performed on bundles of SWNTs, with a distribution of tube diameters $\left(d_{t}\right)$ and chiral angles $(\theta)$. Since the energy values for the van Hove singularities in SWNTs depend on both $d_{t}$ and $\theta$ because of trigonal warping effects, ${ }^{9,10}$ the highly singular DOS expected from theory for one SWNT, cannot be observed experimentally in such a bulk sample. Scanning tunneling spectroscopy (STS) can probe isolated SWNTs, ${ }^{11,12}$ but the features observed by STS, due to van Hove singularities for isolated SWNTs, are normally broad
$\left(\Gamma_{\mathrm{J}} \sim 0.1 \mathrm{eV}\right)$ and do not reflect the extremely high quantum confinement expected from theory, due to the interaction between the STS tip and the single 1D molecule, which perturbs the 1D electronic structure of the SWNT.

RRS has been extensively used to study the 1D characteristics of bulk samples of SWNTs. ${ }^{13}$ Due to the quantum confinement of the DOS, strong resonant effects occur in the Raman scattering from an isolated SWNT when the energy of the incident or scattered light matches an electronic transition $E_{i i}$ between van Hove singularities in the valence and conduction bands, thereby strongly enhancing the Raman signal. $5,6,13,14$

The resonant Raman intensity is proportional to the joint density of electronic states (JDOS). Therefore, the use of the resonant Raman spectra of isolated SWNTs to study their JDOS, and consequently their DOS is theoretically possible, and much effort has therefore been given to measuring such Raman spectra. ${ }^{15-18}$ RRS has an advantage over techniques such as STS, since RRS uses light to probe the DOS of SWNTs and is not expected to significantly perturb their 1D electronic structure. Recently, the production of isolated SWNTs by a chemical vapor deposition (CVD) method ${ }^{19}$ made it possible to measure isolated SWNTs lying on a $\mathrm{Si} / \mathrm{SiO}_{2}$ substrate. ${ }^{20,21}$ Good agreement between the observed radial breathing mode $(\mathrm{RBM})$ frequencies $\left(\omega_{\mathrm{RBM}}\right)$ and theoretical predictions was observed for these SWNTs, based on a proportionality constant of $\alpha=248 \mathrm{~cm}^{-1} \mathrm{~nm}$ in the theoretical dependence of $\omega_{\mathrm{RBM}}$ on SWNT diameter $\left(\omega_{\mathrm{RBM}}\right.$ $\left.=\alpha / d_{t}\right) .{ }^{20}$ The observation of a measurable Raman signal from one SWNT suggests that the intrinsic van Hove singularities in SWNTs must be much sharper than were observed in STS experiments. ${ }^{11,12}$

In this paper, we measure the resonant window for the resonant Raman scattering of one isolated SWNT by using a frequency tunable laser $E_{l}$. We here report an asymmetric 


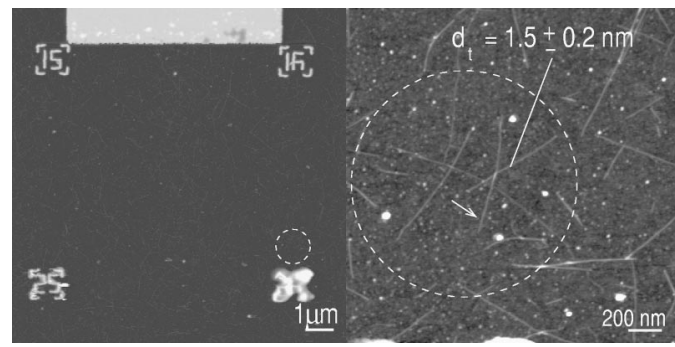

FIG. 1. AFM image of the sample. The left image shows the markers used to localize the spot position (dashed circle) on the substrate during the Raman experiment and for further AFM characterization of the SWNTs present in the light spot (right image).

shape for the $E_{l}$ dependent resonant window, showing the importance of including the detailed experimentally determined JDOS line shape in the resonant Raman theory for 1D systems and considering incoherent scattering. Therefore, we show that RRS can be used to characterize the JDOS of a SWNT with much better resolution than STS measurements.

Isolated SWNTs were prepared by a CVD method on a $\mathrm{Si}$ substrate with a thin $\mathrm{SiO}_{2}$ surface. ${ }^{19,20}$ Figure 1 shows an atomic force microscopy (AFM) image of the substrate with lithographic markers on an $8 \times 8 \mu \mathrm{m}^{2}$ lattice. We chose to put the light spot $(\sim 1 \mu \mathrm{m}$ diameter $)$ close to a mark $(\sim 1 \mu \mathrm{m}$ size) in order to achieve good precision in always returning the light spot to the same position as $E_{l}$ was changed. The dashed circle in Fig. 1 displays the position where we put the laser spot, showing the presence of some isolated SWNTs (see right AFM image of Fig. 1). From the AFM heights, we measured the diameters $\left(d_{t}\right)$ of the 11 SWNTs that lie within the light spot, with $d_{t}$ ranging from $0.7 \mathrm{~nm}$ to $1.9 \mathrm{~nm}$ (the AFM precision is about $\pm 0.2 \mathrm{~nm}$ ). The excitation was provided by a tunable Ti:Sapphire laser $(P$ $<10 \mathrm{~mW}$ on the sample) pumped by an Ar ion laser $(6 \mathrm{~W})$. The incident light was filtered with a single monochromator (Macpherson $1200 \mathrm{~g} / \mathrm{mm}$ ), and the scattered light was analyzed with an XY DILOR triple monochromator equipped with a $\mathrm{N}_{2}$ cooled CCD detector. Raman spectra of the sample were measured in the excitation wavelength (energy) range $720 \mathrm{~nm}(1.722 \mathrm{eV}) \leqslant E_{l} \leqslant 785 \mathrm{~nm}(1.585 \mathrm{eV})$ with steps of $4 \mathrm{~nm}(\sim 0.009 \mathrm{eV})$. All the Stokes and anti-Stokes spectra were corrected to account for spectrometer efficiency at each laser energy, and the spectra were then normalized by the $303 \mathrm{~cm}^{-1}$ Si substrate peak intensities. The anti-Stokes intensities were multiplied by $[n(\omega)+1] / n(\omega)$, where $n(\omega)$ $=1 /\left[\exp \left(\hbar \omega / k_{B} T\right)-1\right]$ is the Bose-Einstein thermal factor, $\omega$ is the frequency, $k_{B}$ is the Boltzmann constant, and $T$ is the temperature. Although we used a high laser power to measure the Raman spectra, $T$ was found to be close to room temperature (not higher than $325 \mathrm{~K}$ ), and this was confirmed by changing the laser power from $1 \mathrm{~mW} / \mu \mathrm{m}^{2}$ $\left(10 \mathrm{MW} / \mathrm{cm}^{2}\right)$ to $10 \mathrm{~mW} / \mu \mathrm{m}^{2}\left(100 \mathrm{MW} / \mathrm{cm}^{2}\right)$, where we found that the Stokes: anti-Stokes intensity ratio for the 521 and $303 \mathrm{~cm}^{-1} \mathrm{Si}$ peaks remained constant, the $\omega_{\text {RBM }}$ peak did not show a temperature dependent shift, and the intensity ratios between the RBM features and the $303 \mathrm{~cm}^{-1}$ Si peaks also remained constant in both the Stokes and anti-Stokes spectra. The gold marker near the light spot, however, prob-

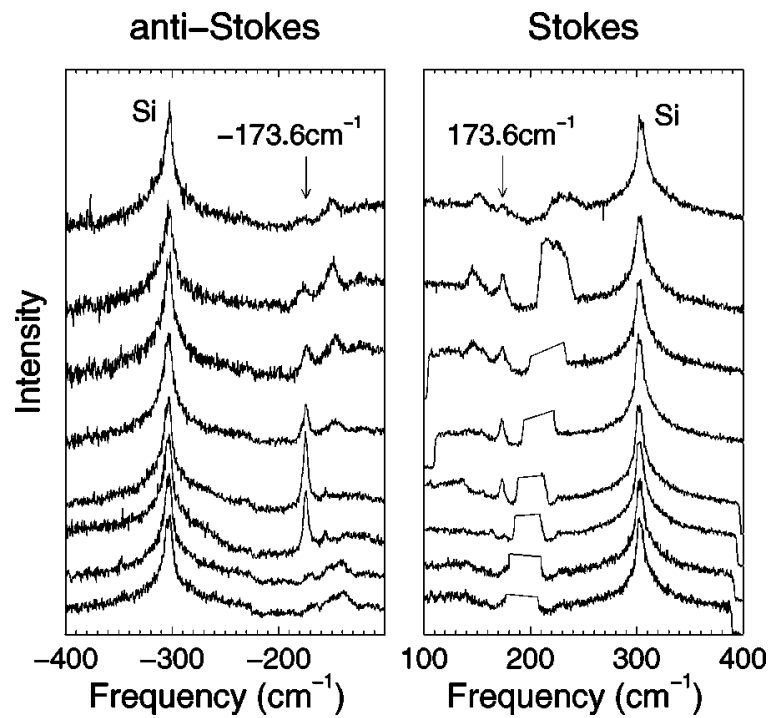

FIG. 2. Stokes and anti-Stokes Raman spectra from isolated SWNTs on a $\mathrm{Si} / \mathrm{SiO}_{2}$ substrate for several different laser excitation energies. From bottom to top, the spectra were taken at $E_{l}=1.623$, $1.631,1.640,1.649,1.666,1.685,1.703$, and $1.722 \mathrm{eV}$. The flat region appearing in all Stokes spectra comes from light leakage, and was cut out from the spectra.

ably experienced some heating.

With the light spot position shown in Fig. 1, we measured the Raman spectra with different laser excitation energies. Figure 2 shows the Stokes and anti-Stokes Raman spectra of one light spot for several different excitation laser energies $E_{l}$ increasing from the bottom to the top spectra (see caption). From Fig. 2, the RBM feature at $173.6 \mathrm{~cm}^{-1}$ appears and disappears over the tunable energy range of $E_{l}$, thereby allowing us to tune over the whole resonant window of one van Hove singularity in the JDOS of this resonant SWNT. The linewidth for this $\omega_{\mathrm{RBM}}=173.6 \mathrm{~cm}^{-1}$ peak is $5 \mathrm{~cm}^{-1}$, typical of one isolated SWNT. ${ }^{20}$ The points in Fig. 3 show the peak intensity of the $173.6 \mathrm{~cm}^{-1} \mathrm{RBM}$ feature vs $E_{l}$ in the Stokes and anti-Stokes processes. As we discuss below, these resonant windows reflect the JDOS of the resonant SWNT.

Figure 3 shows that the resonant window in the antiStokes process is clearly asymmetric, exhibiting, from lower to higher $E_{l}$, a very sharp increase in signal, followed by a slower decrease. The Stokes signal quality is not as good as the anti-Stokes signal due to the frequency dependent spectrometer efficiency that drops off rapidly with increasing laser wavelength, being worse in the Stokes frequency region. However, similar $E_{l}$ dependent behavior is observed for both resonant windows (see Fig. 3). This asymmetric behavior is caused by the asymmetry in the JDOS $g(E)$ of one isolated SWNT. The RBM peak intensity $I\left(E_{l}\right)$, which is a function of $E_{l}$, can be evaluated from $g(E)$ according to

$$
I\left(E_{l}\right)=\int\left|M \frac{g(E)}{\left(E_{l}-E-i \Gamma_{r}\right)\left(E_{l} \pm E_{p h}-E-i \Gamma_{r}\right)}\right|^{2} d E,
$$

where the first and second factors in the denominator, respectively, describe the resonance effect with the incident and 


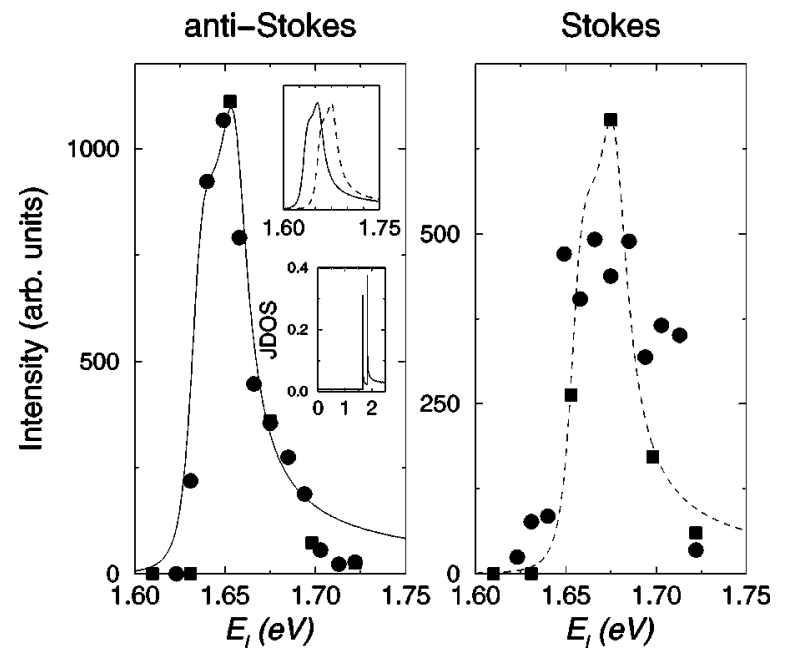

FIG. 3. Raman intensity vs laser excitation energy $E_{l}$ for the $\omega_{\mathrm{RBM}}=173.6 \mathrm{~cm}^{-1}$ peak in the Stokes and anti-Stokes Raman processes. Circles and squares indicate two different $E_{l}$ runs on the same SWNT sample. The line curves indicate the resonant Raman window predicted from Eqs. (1) and (2), with $E_{i i}=1.655 \mathrm{eV}, \Gamma_{r}$ $=8 \mathrm{meV}$, and $\Gamma_{\mathrm{J}}=0.5 \mathrm{meV}$. The upper inset compares the theoretically predicted Stokes and anti-Stokes resonant windows. The lower inset plots the JDOS for one isolated $(18,0) \mathrm{SWNT}$ with $\Gamma_{\mathrm{J}}$ $=0.5 \mathrm{meV}$ for all van Hove singularities up to $2.5 \mathrm{eV}$.

scattered light, where the $+(-)$ applies to the anti-Stokes (Stokes) process for the phonon of energy $E_{p h}, \Gamma_{r}$ gives the inverse lifetime for the resonant scattering process, and $M$ $=M_{i} M_{e p} M_{s}$ is considered to be independent of $E$ in this small energy range, where $M_{i}, M_{s}$, and $M_{e p}$ are, respectively, the matrix elements for the electron-radiation absorption, the electron-radiation emission, and the electronphonon interaction. If the matrix elements for electronic transitions where momentum is not preserved are negligible, and if we assume incoherent scattering, then the matrix $M$ is diagonal, and the square modulus of the Raman tensor can be taken before integrating over $E$.

Since all previous $E_{l}$ dependent studies of RRS were performed on bulk SWNT samples, with a distribution in $d_{t}$ and $\theta$, then $g(E)$ was approximated by a delta function at the maximum JDOS value of the van Hove singularity. ${ }^{6,14,22}$ However, for one isolated SWNT, the detailed JDOS profile must be taken into account, and $g(E)$ can be expressed by

$$
g(E)=\operatorname{Re}\left[\sum_{i} \frac{a_{\mathrm{C}-\mathrm{C}} E}{d_{t} \gamma_{0} \sqrt{\left[\left(E-E_{i i}-i \Gamma_{\mathrm{J}}\right)\left(E+E_{i i}+i \Gamma_{\mathrm{J}}\right)\right.}}\right]
$$

where $a_{\mathrm{C}-\mathrm{C}}$ is the nearest-neighbor distance between carbon atoms, $\gamma_{0}$ is the tight-binding overlap integral, and $\Gamma_{\mathrm{J}}$ is introduced as a measure of the finite nanotube size effect on the width of the JDOS singularity for the $E_{i i}$ electronic transition. The sum over $i$ takes into account the different van Hove singularities of one SWNT.

The line curves in Fig. 3 show plots for the Stokes (dashed line) and anti-Stokes (solid line) resonant windows, using Eqs. (1) and (2) and $E_{p h}=21.5 \mathrm{meV}$ obtained from $\omega_{\text {RBM }}=173.6 \mathrm{~cm}^{-1}$. The width of the resonant windows gives $\Gamma_{r}=8 \mathrm{meV}$, in good agreement with previous measurements. ${ }^{6,7,22} \mathrm{We}$ found $E_{i i}=1.655 \pm 0.003 \mathrm{eV}$ and $\Gamma_{\mathrm{J}}$ in the range of $\sim 0.1-1.0 \mathrm{meV}$, which is much smaller than the widths for the DOS features observed by STS $(\sim 0.1 \mathrm{eV}),{ }^{11,12}$ and accounts for the observed asymmetry in the resonant window of one SWNT. The upper inset shows a comparison between the theoretically predicted Stokes and anti-Stokes resonant windows, revealing a shift in these resonant windows due to the resonant condition for the scattered photon, $E_{l}=E_{i i} \pm E_{p h}$ for the anti-Stokes (+) and the Stokes $(-)$ processes.

From theory, taking $\gamma_{0}=2.90 \mathrm{eV}, a_{\mathrm{C}-\mathrm{C}}=0.144 \mathrm{~nm}$, and $\alpha=248 \mathrm{~cm}^{-1} \mathrm{~nm}$ in the relation $\omega_{\mathrm{RBM}}=\alpha / d_{t},{ }^{20}$ we have very few possibilities for SWNT indices $(n, m)$ which satisfy the observed $E_{i i} \sim 1.655 \mathrm{eV}$ and $\omega_{\mathrm{RBM}} \sim 173.6 \mathrm{~cm}^{-1}$. The best candidates are metallic SWNTs with an $E_{11}^{M}$ that is split by the trigonal warping effect. ${ }^{9,10}$ The indices $(12,9)$ have $d_{t}=1.45 \mathrm{~nm}, \quad E_{11}^{M}=1.684 \mathrm{eV}$ and $1.725 \mathrm{eV}$, and $\omega_{\mathrm{RBM}}$ $=171.2 \mathrm{~cm}^{-1} ;$ indices $(17,2)$ have $d_{t}=1.44 \mathrm{~nm}, E_{11}^{M}$ $=1.650 \mathrm{eV}$ and $1.817 \mathrm{eV}$, and $\omega_{\mathrm{RBM}}=172.7 \mathrm{~cm}^{-1}$; while indices $(18,0)$ have $d_{t}=1.43 \mathrm{~nm}, \quad E_{11}^{M}=1.655 \mathrm{eV}$ and $1.831 \mathrm{eV}$, and $\omega_{\mathrm{RBM}}=173.5 \mathrm{~cm}^{-1}$. Therefore, based on a previously reported method for determining $d_{t}$ and $\theta$ from the measured RBM spectrum ${ }^{20}$ and the $E_{11}^{M}$ value, we identify the $\omega_{\mathrm{RBM}}=173.6 \mathrm{~cm}^{-1}$ signal as coming from a $(18,0)$ SWNT. The experimental value at $E_{i i}=1.655 \mathrm{eV}$ is in very good agreement with the $E_{11}^{M}$ predicted for the $(18,0)$ SWNT based on tight-binding calculations. We did not observe the second van Hove singularity predicted at $1.831 \mathrm{eV}$ because the laser could not be tuned above $1.722 \mathrm{eV}$. Inside the dashed circle in the right image of Fig. 1, there is one SWNT (with $d_{t}=1.5 \pm 0.2 \mathrm{~nm}$ ) which is a good candidate for the resonant $(18,0)$ SWNT. Another possible candidate would be the SWNT with $d_{t}=1.2 \pm 0.2 \mathrm{~nm}$ (see small arrow on the right AFM image of Fig. 1), but this tube is perpendicular to the polarization of the applied electric field, and, due to the antenna effect, ${ }^{17}$ no signal is expected to come from this tube.

Finally, the lower inset to Fig. 3 plots the highly singular JDOS vs $E_{l}$ for one isolated $(18,0)$ SWNT obtained from Eq. (2) and considering the experimental value $\Gamma_{\mathrm{J}}=0.5 \mathrm{meV}$ taken for all van Hove singularities, since $\Gamma_{\mathrm{J}}$ is related to finite-size effects for each SWNT.

In conclusion, by using a tunable laser, it is possible to study the JDOS of one isolated SWNT, giving the $E_{i i}$ value with a precision better than $5 \mathrm{meV}$. The value obtained for $\Gamma_{\mathrm{J}} \sim 0.1-1.0 \mathrm{meV}$ is considerably smaller than the values obtained with STS, ${ }^{11,12}$ suggesting that the DOS in SWNTs exhibits strong 1D behavior. Therefore, resonant Raman spectroscopy can be used to characterize the JDOS of an isolated SWNT, thereby providing important information that could be subsequently used for other properties measurements on this characterized SWNT.

The authors thank Professor K. Kneipp for helpful discussions. The experimental work was performed in the micro- 
Raman laboratory, Universidade Federal de Minas Gerais, Brazil supported by FAPEMIG, CNPq, and FINEP. A.J. and A.G.S.F. acknowledge financial support from the Brazilian agencies CNPq and CAPES, respectively. R.S. acknowl- edges a Grant-in-Aid (Grants No. 11165216 and 13440091) from the Ministry of Education, Japan. The MIT authors acknowledge support under NSF Grant Nos. DMR 9804734, INT 98-15744, and INT 00-00408.
${ }^{1}$ S. Iijima and T. Ichihashi, Nature (London) 363, 603 (1993).

${ }^{2}$ D. S. Bethune, C. H. Kiang, M. S. de Vries, G. Gorman, R. Savoy, J. Vazquez, and R. Beyers, Nature (London) 363, 605 (1993).

${ }^{3}$ M. S. Dresselhaus, G. Dresselhaus, and P. C. Eklund, Science of Fullerenes and Carbon Nanotubes (Academic, New York, NY, 1996).

${ }^{4}$ R. Saito, G. Dresselhaus, and M. S. Dresselhaus, Physical Properties of Carbon Nanotubes (Imperial College, London, 1998).

${ }^{5}$ A. M. Rao, E. Richter, S. Bandow, B. Chase, P. C. Eklund, K. W. Williams, M. Menon, K. R. Subbaswamy, A. Thess, R. E. Smalley, G. Dresselhaus, and M. S. Dresselhaus, Science 275, 187 (1997).

${ }^{6}$ M. A. Pimenta, A. Marucci, S. Empedocles, M. Bawendi, E. B. Hanlon, A. M. Rao, P. C. Eklund, R. E. Smalley, G. Dresselhaus, and M. S. Dresselhaus, Phys. Rev. B 58, R16016 (1998).

${ }^{7}$ M. Milnera, J. Kurti, M. Hulman, and H. Kuzmany, Phys. Rev. Lett. 84, 1324 (2000).

${ }^{8}$ H. Kataura, Y. Kumazawa, N. Kojima, Y. Maniwa, I. Umezu, S. Masubuchi, S. Kazama, X. Zhao, Y. Ando, Y. Ohtsuka, S. Suzuki, and Y. Achiba, Electronic Properties of Novel MaterialsScience and Technology of Molecular Nanostructures, edited by H. Kuzmany, Michael Mehring, Jörg Fink, and Sigmar Roth, AIP Conf. Proc. 486 (AIP, Melville, 1999), 328.

${ }^{9}$ R. Saito, G. Dresselhaus, and M. S. Dresselhaus, Phys. Rev. B 61, 2981 (2000).

${ }^{10}$ P. Kim, T. Odom, J.-L. Huang, and C. M. Lieber, Phys. Rev. Lett. 82, 1225 (1999).
${ }^{11}$ J. W. G. Wildöer, L. C. Venema, A. G. Rinzler, R. E. Smalley, and C. Dekker, Nature (London) 391, 59 (1998).

${ }^{12}$ T. W. Odom, J. L. Huang, P. Kim, and C. M. Lieber, Nature (London) 391, 62 (1998).

${ }^{13}$ M. S. Dresselhaus and P. C. Eklund, Adv. Phys. 49, 705 (2000).

${ }^{14}$ S. D. M. Brown, P. Corio, A. Marucci, M. S. Dresselhaus, M. A. Pimenta, and K. Kneipp, Phys. Rev. B 61, R5137 (2000).

${ }^{15}$ G. S. Duesberg, W. J. Blau, H. J. Byrne, J. Muster, M. Burghard, and S. Roth, Chem. Phys. Lett. 310, 8 (1999).

${ }^{16}$ J. Azoulay, A. Debarre, A. Richard, and P. Tchenio, J. Phys. (France) 10, Pr8-223 (2000).

${ }^{17}$ G. S. Duesberg, I. Loa, M. Burghard, K. Syassen, and S. Roth, Phys. Rev. Lett. 85, 5436 (2000).

${ }^{18}$ K. Kneipp, H. Kneipp, P. Corio, S. D. M. Brown, K. Shafer, J. Motz, L. T. Perelman, E. B. Hanlon, A. Marucci, G. Dresselhaus, and M. S. Dresselhaus, Phys. Rev. Lett. 84, 3470 (2000).

${ }^{19}$ J. H. Hafner, C. L. Cheung, T. H. Oosterkamp, and C. M. Lieber, J. Phys. Chem. B 105, 743 (2001).

${ }^{20}$ A. Jorio, R. Saito, J. Hafner, C. M. Lieber, M. Hunter, T. McClure, G. Dresselhaus, and M. S. Dresselhaus, Phys. Rev. Lett. 86, 1118 (2001).

${ }^{21}$ R. Saito, A. Jorio, J. Hafner, C. M. Lieber, M. Hunter, T. McClure, G. Dresselhaus, and M. S. Dresselhaus, Phys. Rev. B (unpublished).

${ }^{22}$ P. M. Rafailov, H. Jantoliak, and C. Thomsen, Phys. Rev. B 61, 16179 (2000) 